\title{
Quercetin exerts a neuroprotective effect through inhibition of the iNOS/NO system and pro-inflammation gene expression in PC12 cells and in zebrafish
}

\author{
ZAI JUN ZHANG ${ }^{1,2}$, LORITA CHI VENG CHEANG ${ }^{1}$, MEI WEI WANG ${ }^{1}$ and SIMON MING-YUEN LEE ${ }^{1}$ \\ ${ }^{1}$ Institute of Chinese Medical Sciences, University of Macau, Av. Padre Toma's Pereira S.J., Taipa, Macao SAR; \\ ${ }^{2}$ Institute of New Drug Research and Guangdong Province Key Laboratory of Pharmacodynamic \\ Constituents of Traditional Chinese Medicine and New Drug Research, Jinan University \\ College of Pharmacy, Guangzhou, P.R. China
}

Received July 21, 2010; Accepted October 1, 2010

DOI: $10.3892 / \mathrm{ijmm} .2010 .571$

\begin{abstract}
Flavonoids have been reported to be potent antioxidants and beneficial in the treatment of oxidative stress-related diseases. Quercetin, a major flavonoid naturally occurring in plants, deserves attention because of its beneficial effects observed in various in vitro and in vivo neural damage models; however, the actions of quercetin are paradoxical. In an effort to confirm the neuroprotective effect of quercetin and to elucidate its mechanism of action, the neuroprotective effects of quercetin in PC12 cells and in zebrafish models were investigated. In this study, the selective dopaminergic neurotoxin 6-hydroxydopamine (6-OHDA), was used to induce neural damage in PC12 cells and zebrafish models. Pretreatment with quercetin offered neuroprotection against 6-OHDA-induced PC12 cell death. Moreover, quercetin could prevent 6-OHDA-induced PC12 cell apoptosis and 6-OHDA-stimulated dopaminergic neuron loss in zebrafish. Interestingly, quercetin was able to protect, but not rescue the dopaminergic neuron damage when zebrafish were treated with quercetin at different maturation stages of the blood brain barrier. A mechanistic study showed that quercetin could inhibit NO over-production and iNOS over-expression in PC12 cells and could down-regulate the over-expression of pro-inflammatory genes (e.g. IL-1ß, TNF- $\alpha$ and COX-2) in zebrafish, suggesting that these genes play a role in the neuroprotective effect of quercetin. The objective of this study was to provide a scientific rationale for the clinical use of quercetin, leading to its development as an effective therapeutic agent for the treatment of Parkinson's disease.
\end{abstract}

Correspondence to: Dr Simon Ming-Yuen Lee, Institute of Chinese Medical Sciences, University of Macau, Av. Padre Toma's Pereira S.J., Taipa, Macao SAR, P.R. China

E-mail: simonlee@umac.mo

Key words: quercetin, Parkinson's disease, neuroprotection, zebrafish

\section{Introduction}

Parkinson's disease (PD) is the second most common neurodegenerative disease with physiological manifestations including tremors, bradykinesia, abnormal postural reflexes, rigidity and akinesia. Although the etiology of PD is not completely understood, increasing evidence suggests that oxidative damage induced by reactive oxygen species (ROS) and reactive nitrogen species (RNS) is involved in the progression of dopaminergic neurodegeneration $(1,2)$. As a result, inhibition of ROS and of nitric oxide (NO) and iNOS production, serve as potential targets for protection against PD pathogenesis.

Increasing evidence suggests that neuro-inflammatory processes contribute to the cascade leading to the progressive neuronal damage in PD (3). Activation of glial cells, such as astrocytes and microglia also plays a key role in the development of inflammatory neurodegeneration (4). The major intermediate of glial-induced neurotoxicity is the generation of NO via increased expression of iNOS. Excessive NO can induce neuronal cell damage by disrupting the function of the neuronal mitochondrial electron transport chain (5). Inflammation is also characterized by increased production of cytokines, such as IL- $1 \beta$ and TNF- $\alpha$, which also act to stimulate iNOS and NO production (6). Hence, modulation of NO production, glial signalling cascades and pro-inflammatory cytokines might result in suppression of neuro-inflammation and may ultimately protect against neurodegeneration. Although the neuroprotective effects of quercetin are found to be via its anti-oxidative and antiinflammatory capacity in an in vitro system of lipopolysaccahride-activated co-culture of microglial and neuronal cells $(7,8)$, it is uncertain whether its mode of action in the in vivo $\mathrm{PD}$ model involves anti-inflammation.

Quercetin, which is one of the most common naturally occurring flavonoids, has been shown to possess diverse biological activities, including anti-tumoral, anti-thrombotic, anti-inflammatory and anti-apoptotic effects (9-12). Although quercetin and structurally related flavonoids have been shown to have a neuroprotective capacity in various in vitro and in vivo experimental models (13-16), the neuroprotective 
effect of quercetin remains controversial (17). Nevertheless, quercetin did not protect substantia nigra neurons from an oxidative insult in vivo, probably due to its inability to cross the blood-brain barrier (BBB) under in vivo conditions (18). There is an urgent need for appropriate in vivo studies in order to confirm the neuroprotective effects of quercetin and to identify the reason for the discrepancy between earlier in vitro and in vivo findings.

The zebrafish (Danio rerio) has become a powerful model for drug discovery $(19,20)$. Its rapid development and rather simple, short assay time make zebrafish a useful tool in largescale drug screening (21). The brain structure and function of the zebrafish are very similar to those of other vertebrates (22). The anatomy of the zebrafish brain dopaminergic (DA) system was studied recently, and a region anatomically similar to the striatum was identified in the forebrain (23). Most neurotoxins, such as MPTP, 6-hydroxydopamine (OHDA) and rotenone, are known to induce DA neuron loss in animal models. Zebrafish PD models have been well validated and zebrafish develop relevant $P D$ pathological conditions in response to these toxins (24-26). Moreover, exposure of zebrafish to neurotoxins induces relevant clinical behavioural features of PD reflected by reduced swimming velocity and total distance moved. In addition, those clinical and experimental neuroprotective agents have been demonstrated to have equivalent physiological activity in protecting zebrafish from neuronal insult $(27,28)$. Taken together, the results of these earlier studies suggest that the zebrafish is a good alternative PD model and offers great opportunity for screening and discovery of novel PD therapeutic agents. In order to address the above-mentioned controversy concerning the use of quercetin for the treatment of PD and the lack of details about the anti-neuro-inflammatory action of quercetin in vivo, the neuroprotective effect and the underlying mechanism of action of quercetin were investigated using 6-OHDA-treated zebrafish and PC12 cells in this study.

\section{Materials and methods}

Cell culture. Stock cultures of rat pheochromocytoma cells (PC12) were purchased from the American Type Culture Collection (ATCC, Manassas, VA, USA). They were cultured in a humidified atmosphere of $95 \%$ air and $5 \% \mathrm{CO}_{2}$ at $37^{\circ} \mathrm{C}$ in F-12K medium supplemented with $15 \%(\mathrm{v} / \mathrm{v})$ heat-inactivated horse serum, $2.5 \%(\mathrm{v} / \mathrm{v}) \mathrm{FBS}$, penicillin $(100 \mathrm{U} / \mathrm{ml})$ and streptomycin $(100 \mu \mathrm{g} / \mathrm{ml})$, which was changed every other day.

3-(4,5-Dimethyl-2-thiazolyl)-2,5-diphenyl-2H-tetrazolium bromide (MTT) assay. MTT is a tetrazolium salt that can be reduced to purple formazan by living cells. Cells were incubated for $4 \mathrm{~h}$ at $37^{\circ} \mathrm{C}$ in MTT solution (prepared in fresh $0.5 \%(\mathrm{v} / \mathrm{v})$ heat-inactivated horse serum; final concentration $0.5 \mathrm{mg} / \mathrm{ml})$. The medium was then discarded and $150 \mu \mathrm{l}$ of DMSO was added to the well to dissolve the violet formazan crystals in intact cells. The absorbance was measured at a wavelength of $490 \mathrm{~nm}$ by a multilabel counter (Wallac Victor $3^{\mathrm{TM}} \mathrm{V}$, Perkin-Elmer, The Netherlands). Cell viability was expressed as a percentage of the control (untreated cells). All assays were done in 8 replicates and at least 3 times.
Lactate dehydrogenase $(L D H)$ assay. Cell viability was determined by measuring the activity of $\mathrm{LDH}$ released into the incubation medium when cellular membranes are damaged. Cells were plated in 96-well plates and the amount of $\mathrm{LDH}$ released was measured and then the cells were lysed to measure the total $\mathrm{LDH}$. Total and released $\mathrm{LDH}$ activities were determined according to the instructions accompanying the Cytotoxicity Detection Kit (Roche). Absorbance was measured with an automatic microplate reader at $490 \mathrm{~nm}$. LDH released was normalized to total LDH and the results are shown as a percentage of the control value.

Intracellular NO detection. Intracellular NO was evaluated by using the fluorescent probe 4-amino-5-methylamino2',7'-difluorofluorescein diacetate (DAF-FM diacetate), which is cell-permeant and diffuses passively across cellular membranes. Once inside the cells, DAF-FM diacetate is deacetylated by intracellular esterases to DAF-FM, which is essentially non-fluorescent until it reacts with NO; thus, it can be used to quantify intracellular NO production. The cells were seeded in 96-well, black bottom-clear plates. After treatment with quercetin for $6 \mathrm{~h}$ and then exposure to 6-OHDA for $1 \mathrm{~h}$, cells were washed in PBS and incubated for $20 \mathrm{~min}$ at $37^{\circ} \mathrm{C}$ in darkness in a medium containing $1 \%$ serum plus $2.5 \mu \mathrm{M}$ DAF-FM diacetate. The cells were then washed twice in PBS and the fluorescence was evaluated in a microplate reader at an excitation wavelength of $495 \mathrm{~nm}$ and an emission wavelength of $515 \mathrm{~nm}$. The increase in fluorescence for each treatment was calculated as the relative fluorescence of each treatment compared with the untreated control cells.

Western blot analysis. After treatment, PC12 cells were washed three times with PBS and then RIPA lysis buffer containing 1\% PMSF and 1\% Protease Inhibitor Cocktail and incubated for $30 \mathrm{~min}$ on ice. Cell lysates were centrifuged at $12,500 \mathrm{x} \mathrm{g}$ for $20 \mathrm{~min}$ at $4^{\circ} \mathrm{C}$. The supernatant was separated and the amount of protein was determined using the BCA protein assay kit. Protein samples $(30 \mu \mathrm{g})$ were separated by SDS-PAGE (12\% (w/v) polyacrylamide gel) and then transferred to a polyvinylidene difluoride (PVDF) membrane (Bio-Rad, Hercules, CA). Subsequently, the membrane was blocked with 5\% (v/v) non-fat milk in PBST (PBS containing $0.1 \%$ Tween-20) for $1 \mathrm{~h}$ at room temperature. The blots were incubated overnight at $4{ }^{\circ} \mathrm{C}$ with primary antibodies anti-iNOS and anti- $\beta$-actin $(1: 1000$, from Cell Signaling, Inc.). $\beta$-actin was used as the internal reference. After three washes with PBST, the membranes were incubated with horseradish peroxidase-conjugated secondary antibodies (1:2000) in PBST with $5 \%$ non-fat milk for $1 \mathrm{~h}$ at room temperature. After repeated washes, proteins were visualized with an ECL advanced Western blotting detection kit (Amersham, UK) according to the manufacturer's protocol. Photographs of protein bands were taken by the Molecular Imager ChemiDoc XRS (Bio-Rad). Quantitative assessment of protein bands was done with Gel Doc ${ }^{\mathrm{TM}}$ XRS equipped with Quantity One Software.

Fish maintenance. The AB wild-type strain of zebrafish was used for this study. Embryos were collected after natural spawning, staged according to standard criteria, and raised 

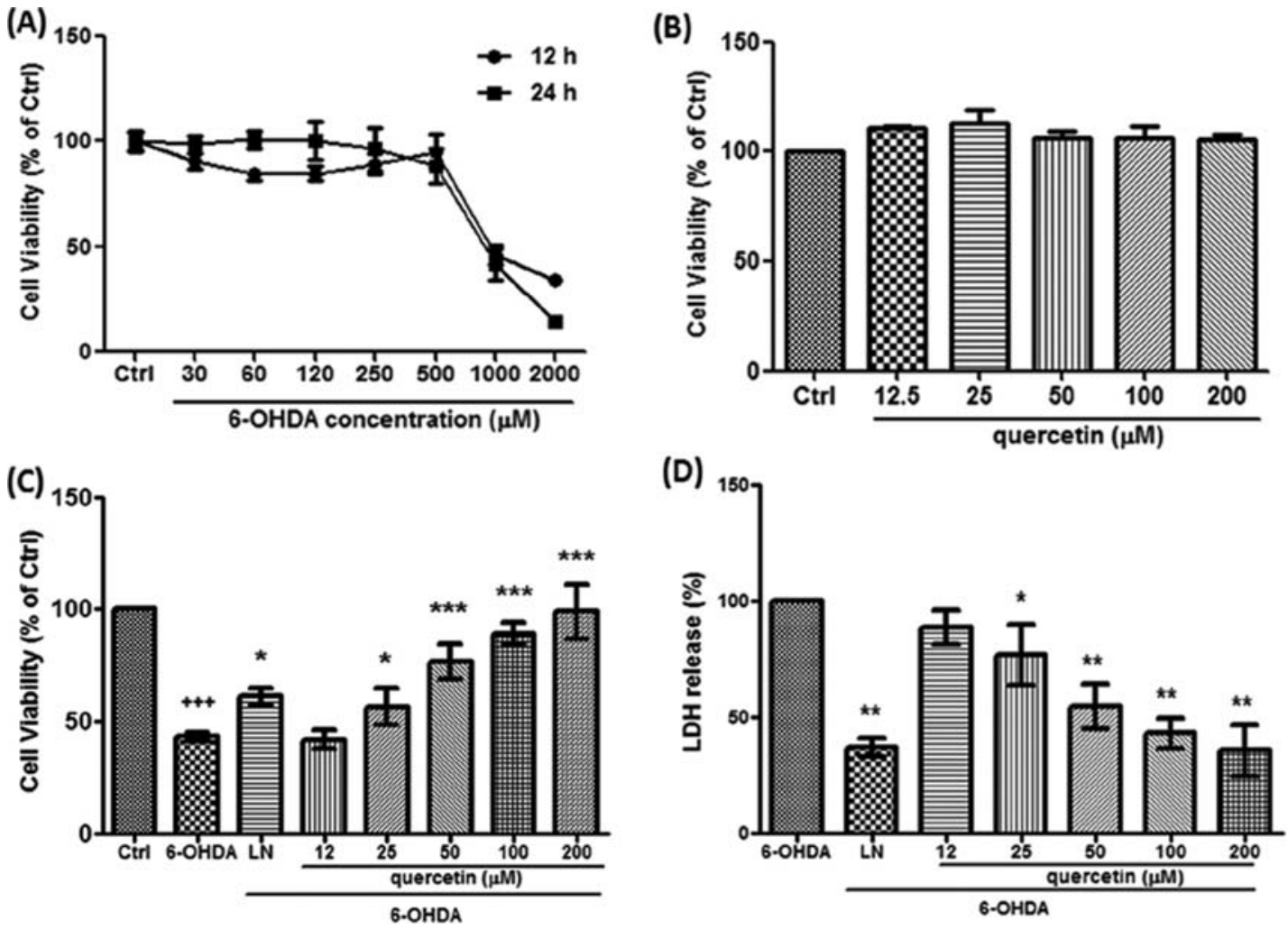

Figure 1. Quercetin dose-dependent inhibition of 6-hydroxydopamine (6-OHDA)-induced cell death in PC12 cells. (A) PC12 cells were incubated for $12 \mathrm{~h}$ or $24 \mathrm{~h}$ with different concentrations of 6-OHDA. (B) Cells were treated for $6 \mathrm{~h}$ with or without different concentrations of quercetin. (C, D) Cells were pretreated for $6 \mathrm{~h}$ with or without different concentrations of quercetin. Cells pretreated for $6 \mathrm{~h}$ with $250 \mu \mathrm{M} \mathrm{L-NAME} \mathrm{(LN)} \mathrm{served} \mathrm{as} \mathrm{a} \mathrm{positive} \mathrm{control.} \mathrm{All}$ groups were exposed to $1 \mathrm{mM}$ 6-OHDA for another $12 \mathrm{~h}$ after the pretreatment. In A-C, cell viability was measured by the MTT assay and the results are expressed as a percentage of that of the control group (without treatment with 6-OHDA). (D) Same as (C) and (D), except the degree of cell death was measured as the percentage of released lactate dehydrogenase (LDH). ${ }^{++} \mathrm{p}<0.0001 \mathrm{vs.}$ the control group; ${ }^{*} \mathrm{p}<0.05,{ }^{* *} \mathrm{p}<0.01$ and ${ }^{* * *} \mathrm{p}<0.0001 \mathrm{vs}$. the $6-\mathrm{OHDA}$ group. Each experiment in the figure was done at least in triplicate.

synchronously at $28.5^{\circ} \mathrm{C}$ in embryo medium $(13.7 \mathrm{mM} \mathrm{NaCl}$, $540 \mu \mathrm{M} \mathrm{KCl}, \mathrm{pH} 7.4,25 \mu \mathrm{M} \mathrm{Na}_{2} \mathrm{HPO}_{4}, 44 \mu \mathrm{M} \mathrm{KH}_{2} \mathrm{PO}_{4}$, $300 \mu \mathrm{M} \mathrm{CaCl}_{2}, 100 \mu \mathrm{M} \mathrm{MgSO}_{4}, 420 \mu \mathrm{M} \mathrm{NaHCO}_{3}, \mathrm{pH}$ 7.4). No additional maintenance was required because the embryos receive nourishment from the attached yolk ball. Ethical approval for the animal experiments was granted by the Animal Research Ethics Committee, University of Macau.

Anti-tyrosine hydroxylase (TH) whole-mount immunostaining. Zebrafish were fixed in 4\% paraformaldehyde in PBS for $5 \mathrm{~h}$, rinsed, and stored at $-20^{\circ} \mathrm{C}$ in $100 \% \mathrm{EtOH}$. Whole-mount immunostaining was done by standard methods (29). Briefly, fixed samples were blocked (2\% lamb serum and $0.1 \%$ BSA in PBST) for $1 \mathrm{~h}$ at room temperature. A mouse monoclonal anti-TH antibody (1:200 diluted in blocking buffer, MAB318, Millipore) was used as the primary antibody and incubated with the sample overnight at $4^{\circ} \mathrm{C}$. The next day, samples were washed 6 times with PBST (30 min each wash), followed by incubation with secondary antibody according to the instructions of the Vectastain ABC kit (Vector Laboratories, Inc.). After staining, zebrafish were flat-mounted with $3.5 \%$ methylcellulose and photographed.

Total RNA extraction and reverse transcription and quantitative real-time PCR. Zebrafish embryos at $48 \mathrm{hpf}$ were treated for $24 \mathrm{~h}$ with different concentrations of quercetin in the presence or absence of 6-OHDA. Total RNA was extracted from 40 zebrafish embryos of each treatment group using the RNeasy Mini Kit (Qiagen, USA) in accordance with the manufacturer's instructions. RNA was reverse transcribed to single-stranded cDNA using the SuperScript ${ }^{\mathrm{TM}}$ III FirstStrand Synthesis System for RT-PCR (Invitrogen, USA), followed by real-time PCR using the TaqMan ${ }^{\circledR}$ Universal PCR Master Mix and TaqMan ${ }^{\circledR}$ gene expression assay primer for zebrafish IL-1ß (assay ID Dr03114368_m1), TNF- $\alpha$ (assay ID Dr03126849_g1) and COX-2 (assay ID Dr03080323_m1) in the ABI 7500 real-time PCR system (all products from Applied Biosystems). The expression level of each gene was expressed as the relative fold change calculated using the comparative CT method (30) and $B$-actin was used as the internal reference.

Statistics. One-way ANOVA and the Dunnett's test were used to identify significant differences between treatment groups and the vehicle control. All calculations were done using the GraphPad Prism statistical software (GraphPad Software, Inc., San Diego, CA) and the level of statistical significance was set at $\mathrm{p}<0.05$.

\section{Results}

Quercetin protects PC12 cells against 6-OHDA-induced cytotoxicity. 6-OHDA-induced cellular damage was evaluated by determining the percentage of MTT reduction in PC12 

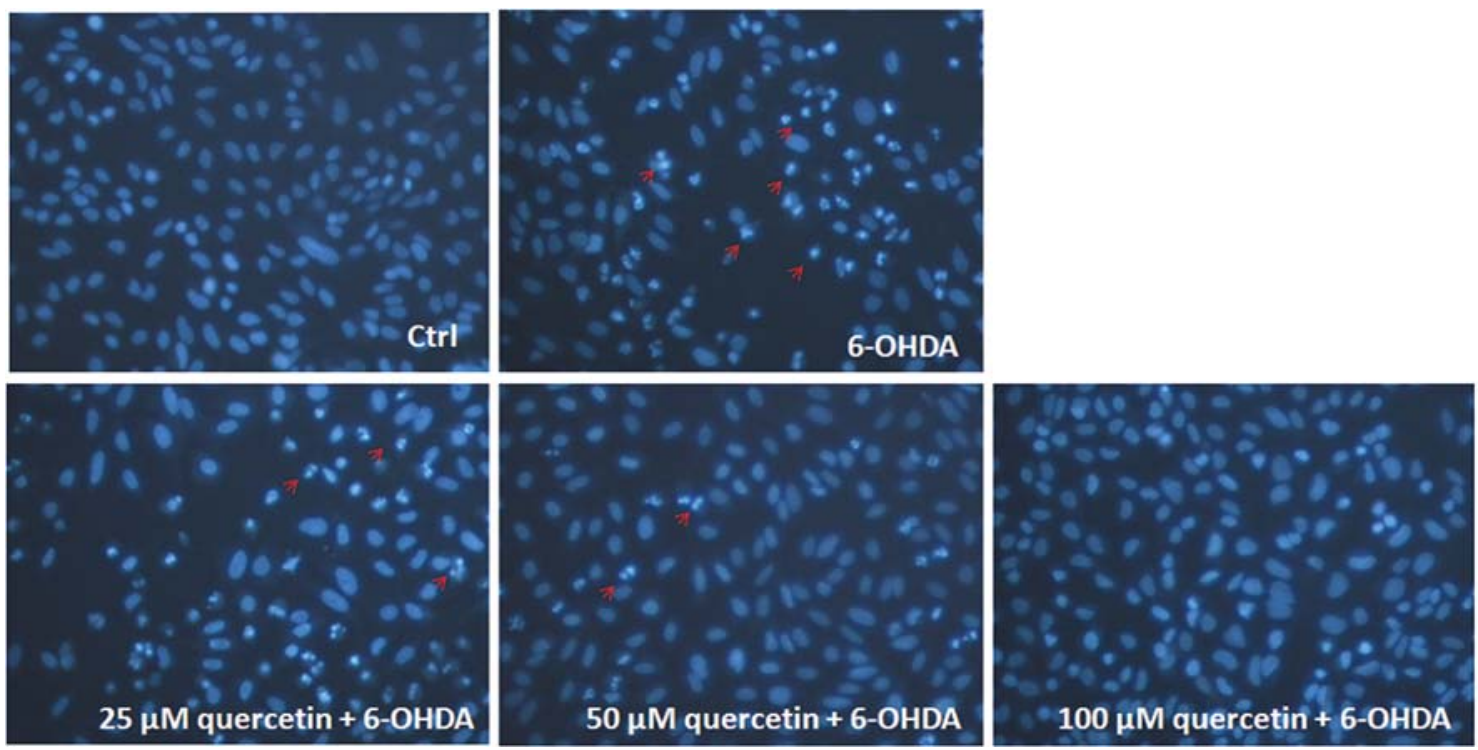

Figure 2. Anti-apoptotic effect of quercetin against 6-hydroxydopamine (6-OHDA)-induced damage to PC12 cells. PC12 cells were treated for $6 \mathrm{~h}$ with or without different concentrations of quercetin, then exposed to $1 \mathrm{mM}$ 6-OHDA for another $12 \mathrm{~h}$. Cells were stained with the DNA-binding fluorescent dye Hoechst 33342. Chromatin condensation and DNA fragments are indicated by the red arrows. Magnification x400.

(A)
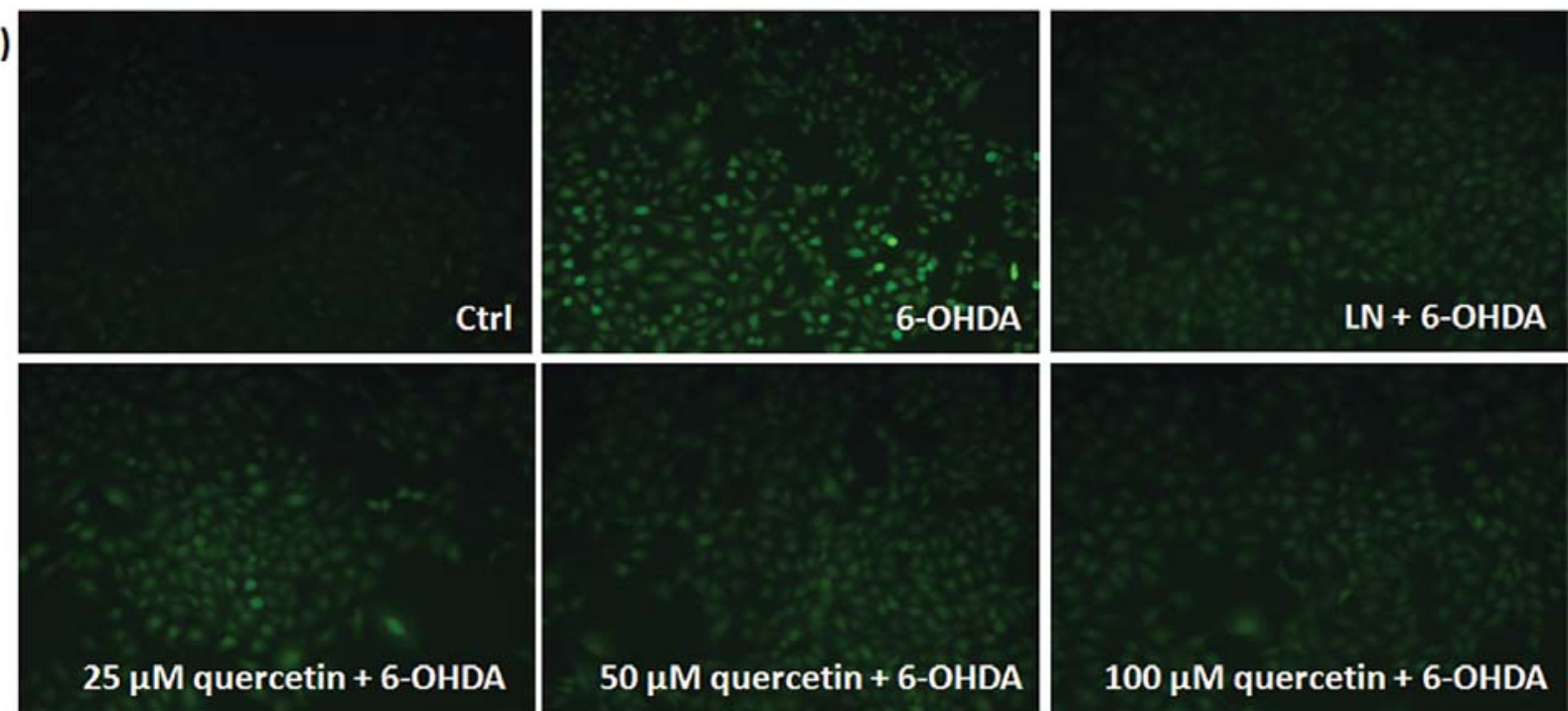

(B)

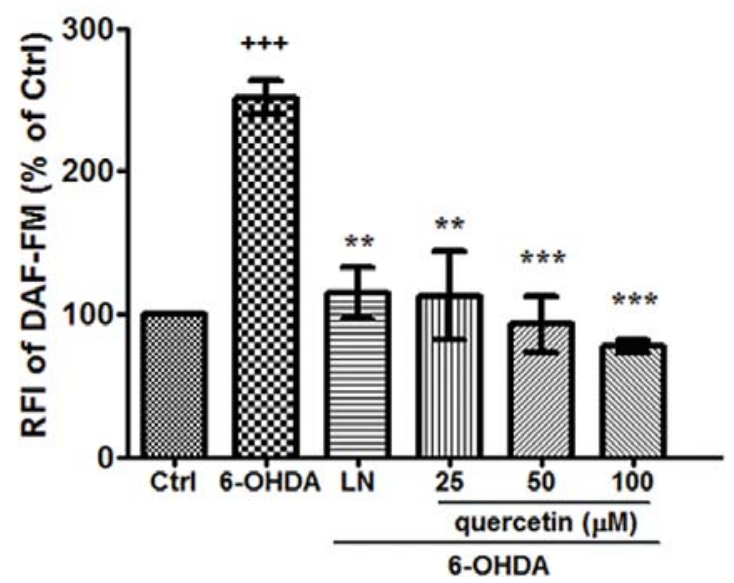

Figure 3. Quercetin-inhibited 6-hydroxydopamine (6-OHDA)-induced nitric oxide (NO) over-production in PC12 cells. PC12 cells were treated for 6 h with or without different concentrations of quercetin, then exposed to $1 \mathrm{mM}$ 6-OHDA for $1 \mathrm{~h}$. (A) Intracellular NO was stained by the fluorescent indicator DAF-FM diacetate. (B) The NO fluorescent intensity was quantified by a multilabel counter. Results are expressed as a percentage of relative fluorescent intensity (RFI) of the control (Ctrl) group. ${ }^{++} \mathrm{p}<0.0001$ vs. the control group; ${ }^{* *} \mathrm{p}<0.01$ and ${ }^{* * *} \mathrm{p}<0.0001 \mathrm{vs}$. the 6-OHDA group. Magnification $\mathrm{x} 200$. 
cells. As shown in Fig. 1A, 6-OHDA caused a dose-dependent reduction of $\mathrm{PC} 12$ cell viability. When $\mathrm{PC} 12$ cells were exposed to $1 \mathrm{mM}$ 6-OHDA for $12 \mathrm{~h}$, cell viability was reduced significantly to $46.2 \pm 4.5 \%$ of the control group viability. Cytotoxicity evaluation indicated that quercetin did not impose any cytotoxicity on PC12 cells up to the maximum concentration of $200 \mu \mathrm{M}$ (Fig. 1B). In order to study the neuroprotective effects of quercetin, $\mathrm{PC} 12$ cells were treated with different concentrations $(12.5,25,50,100$ and $200 \mu \mathrm{M})$ of quercetin for $6 \mathrm{~h}$ before exposure to $1 \mathrm{mM}$ 6-OHDA for $12 \mathrm{~h}$. The protective effects of quercetin were determined by the MTT and LDH assays. As shown in Fig. 1C, pretreatment with quercetin protected PC12 cells against 6-OHDAinduced damage in a dose-dependent manner. Similarly, pretreatment with quercetin significantly reduced $(\mathrm{p}<0.05)$ the LDH leakage caused by 6-OHDA (Fig. 1D). L-NAME $(\mathrm{LN})$ is an iNOS inhibitor that protects PC12 cells against 6-OHDA cytotoxicity by inhibiting 6-OHDA-induced NO over-production and served as the positive control in this study (31). In the present study, the protective effect of quercetin was even stronger than that of LN.

Quercetin protects PC12 cells against 6-OHDA-induced apoptosis. In order to investigate whether 6-OHDA induces cell death through apoptosis, DNA staining with Hoechst 33342 was used to evaluate chromatin condensation and nuclear fragmentation in PC12 cells. As shown in Fig. 2, normal cells were circular or elliptical with no nuclear condensation or fragmentation. In contrast, bright condensed dots known as apoptotic bodies (Fig. 2, red arrows) were clearly identified in 6-OHDA-treated PC12 cells and reductions in colony density and cell size were observed. However, 6-OHDA-induced nuclear condensation was attenuated significantly by pretreatment with quercetin (Fig. 2, lower panel). Moreover, higher quercetin concentrations (50 and $100 \mu \mathrm{M}$ ) inhibited 6-OHDA-induced colony reduction and cell shrinkage.

Quercetin attenuates 6-OHDA-induced NO over-production and iNOS over-expression in PC12 cells. Augmented NO production subsequent to iNOS induction appears to play an important role in the initial phase of 6-OHDA-induced neuro-damage models in vitro and in vivo (32-34). We tested the effect of quercetin on 6-OHDA-induced NO overproduction and iNOS over-expression in PC12 cells. Stimulation of PC12 cells with $1 \mathrm{mM}$ 6-OHDA for $1 \mathrm{~h}$ caused a significant, almost 3 -fold, increase compared to untreated cells (Fig. 3). Because 6-OHDA-induced NO overproduction decreased when treatment with 6-OHDA was for longer than $1 \mathrm{~h}$ (data not shown), we incubated PC12 cells with 6-OHDA for $1 \mathrm{~h}$ when investigating the NO-inhibitory effect of quercetin. As shown in Fig. 3A and B, pretreatment with quercetin for $6 \mathrm{~h}$ inhibited 6-OHDA-induced NO overproduction in a concentration-dependent manner and the level of NO was attenuated to normal (control group with no 6-OHDA treatment) by high concentrations (50 and $100 \mu \mathrm{M}$ ) of quercetin. Moreover, the NO-inhibitory effect of high concentrations of quercetin $(50$ and $100 \mu \mathrm{M})$ was stronger than that in the positive control, which used $250 \mu \mathrm{M} \mathrm{LN}$, a non-selective iNOS inhibitor (Fig. 3). These results are, in part, supportive of our finding shown in Fig. 1, that quercetin exhibits stronger protective effects compared to $\mathrm{LN}$ against 6-OHDA-induced damage in PC12 cells.

Furthermore, Western blot analysis revealed that stimulation of PC12 cells with 6-OHDA for $30 \mathrm{~min}$ and $1 \mathrm{~h}$ significantly enhanced iNOS protein expression (Fig. 4A and C). However, the enhanced iNOS expression started to diminish when the cells were treated with 6-OHDA for longer than $1 \mathrm{~h}$. The time-dependent response of iNOS expression to treatment with 6-OHDA was correlated with that of NO production (Fig. 3). Pretreatment with quercetin also results in a concentration-dependent down-regulation of 6-OHDA-induced iNOS over-expression, similar to the inhibitory effect observed in 6-OHDA-induced NO overproduction. This implies that quercetin attenuated NO overproduction via down-regulation of iNOS over-expression in 6-OHDA-treated PC12 cells.

Quercetin can prevent, but not rescue the DA neuron loss induced by 6-OHDA in zebrafish. The development of DA neurons in zebrafish is similar to that in other vertebrates and thus the zebrafish is a good model to study disorders of the DA system (23). To further investigate the neuroprotective effect of quercetin in vivo, we examined the DA neurons in zebrafish by whole-mount immunostaining with an antibody against $\mathrm{TH}$, the rate-determining enzyme involved in the synthesis of DA. As shown in Fig. 5B and C, the diencephalic DA neurons became matured at $3 \mathrm{dpf}$. When compared with the control, exposure of $1 \mathrm{dpf}$ zebrafish to 6-OHDA for $48 \mathrm{~h}$ results in about $50 \%$ reduction of $\mathrm{TH}$-positive neurons in the diencephalic area of the zebrafish embryo. Co-treatment with quercetin (6 or $12 \mu \mathrm{M})$ significantly inhibited 6-OHDAinduced DA neuron loss by approximately $20 \%$ compared with the 6-OHDA group (Fig. 5B). Nomifensine, a DAT inhibitor, was used as the positive control and was shown to exert considerable protection against 6-OHDA-induced DA neuron loss (Fig. 5B). However, quercetin could not reverse the 6-OHDA-induced DA neuron loss if it was administered after a $48 \mathrm{~h}$ exposure to 6-OHDA (Fig. 6).

Quercetin reverses 6-OHDA-induced pro-inflammatory gene over-expression in zebrafish. Neuro-inflammation plays a key role in 6-OHDA-induced DA neuron damage in vivo (35). We used quantitative real-time PCR to measure inflammationrelated gene expression in 6-OHDA-treated zebrafish. As shown in Fig. 7, 6-OHDA caused over-expression of IL-1ß, TNF- $\alpha$ and COX-2, several fold greater than that of untreated control fish. Co-treatment with quercetin reversed the upregulated inflammatory gene expression, revealing that the anti-inflammatory activity of quercetin is involved in its neuroprotective effect in the zebrafish model of 6-OHDAinduced neuronal damage.

\section{Discussion}

The present study showed that quercetin is a potent neuroprotective agent in both PC12 cells in vitro and zebrafish in vivo. Furthermore, we have shown that its neuroprotective activity might be exerted via the inhibition of NO overproduction by down-regulating the over-expression of iNOS 
(A)

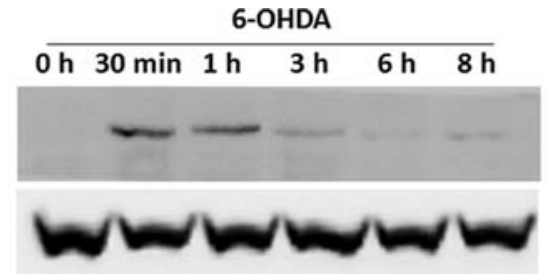

(C)

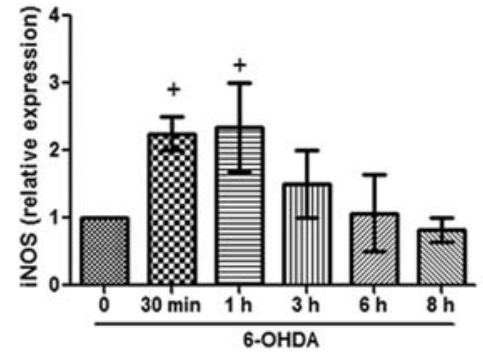

(B)

\section{Ctrl 6-OHDA Que 25 Que 50 Que100}

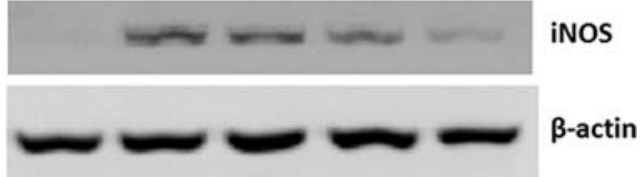

(D)

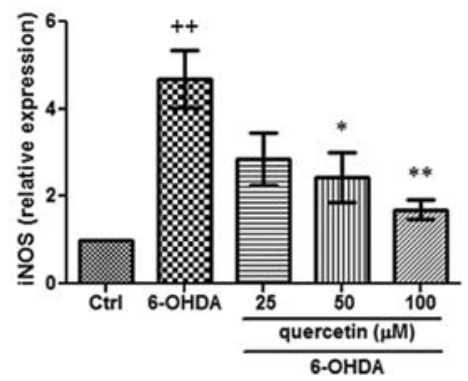

Figure 4. Quercetin down-regulates iNOS over-expression in PC12 cells stimulated by 6-hydroxydopamine (6-OHDA). (A, C) PC12 cells were treated with $1 \mathrm{mM}$ 6-OHDA for $30 \mathrm{~min}, 1,3,6$ and $8 \mathrm{~h}$. iNOS expression was evaluated at these time points. (B, D) PC12 cells were treated for $6 \mathrm{~h}$ with quercetin (Que), then exposed to $1 \mathrm{mM}$ 6-OHDA for another $1 \mathrm{~h}$. Quercetin dose-dependent inhibition of 6-OHDA-induced iNOS over-expression. ${ }^{+} \mathrm{p}<0.05 \mathrm{vs} .0 \mathrm{~h}$ group (before treatment with 6-OHDA); ${ }^{++} \mathrm{p}<0.01$ vs. the control group; ${ }^{\mathrm{p}}<0.05$ and ${ }^{* *} \mathrm{p}<0.01$ vs. the 6-OHDA-treated group.

(A)

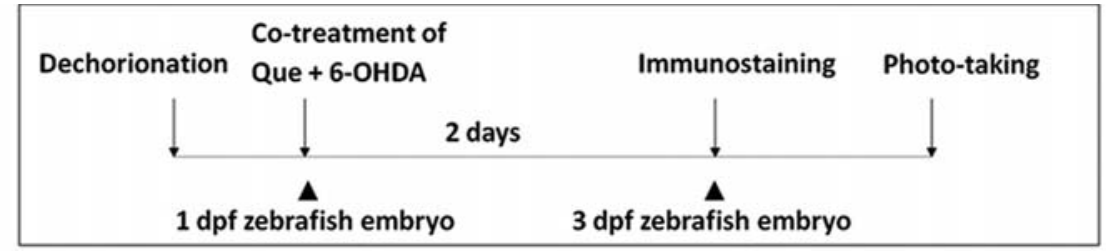

(B)
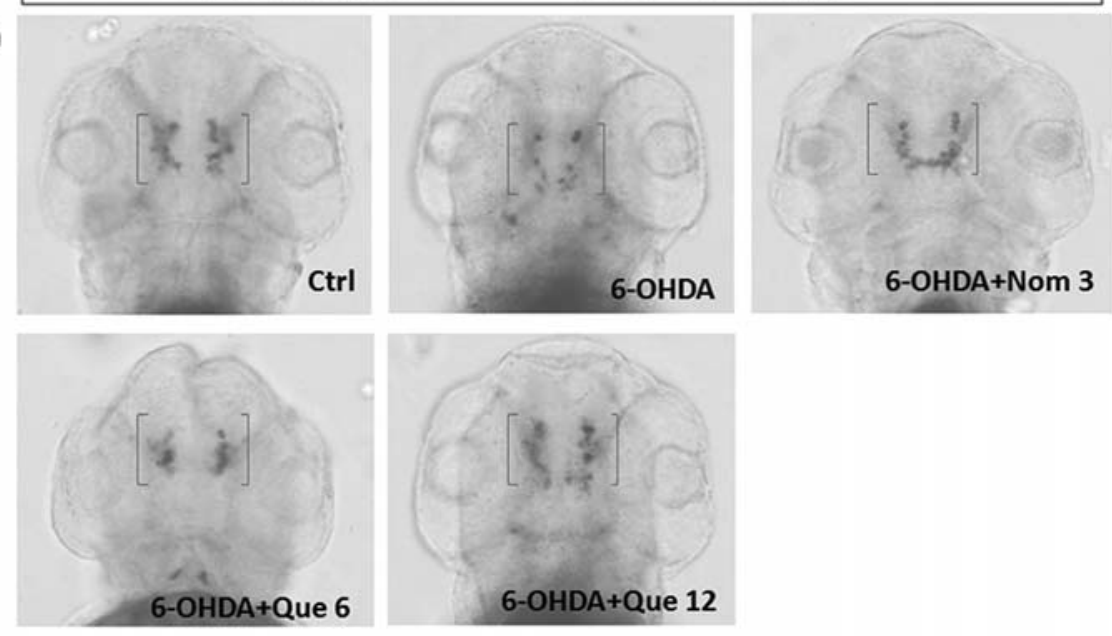

(C)

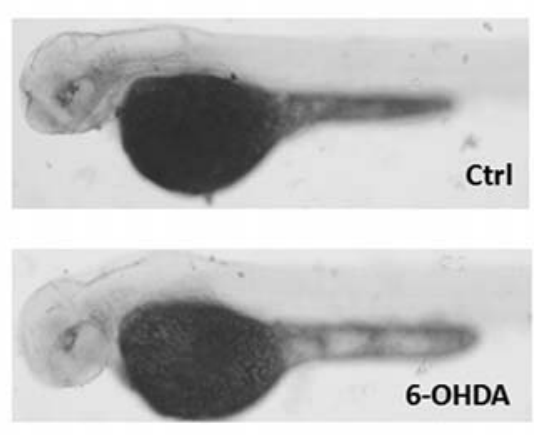

(D)

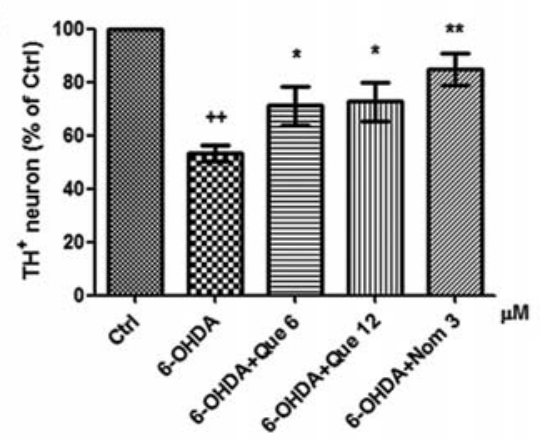

Figure 5. Quercetin protects against the 6-hydroxydopamine (6-OHDA)-induced dopaminergic neuron loss in zebrafish. (A) A schematic illustration of drug treatment. $1 \mathrm{dpf}$ dechorionated zebrafish were treated for $48 \mathrm{~h}$ with $250 \mu \mathrm{M}$ 6-OHDA in the presence or absence of quercetin (Que) or nomifensine (Nom, a DAT inhibitor used as a positive control). After treatment, anti-tyrosine hydroxylase (TH) immunostaining was used to detect the DA neurons in zebrafish. (B and C) Representative morphology of DA neurons in zebrafish brain. The diencephalic DA neurons (Di DA) are indicated by brackets. (B) Ventral view, head at the top; (C) lateral view, head at the left. (D) Quantitative analysis of $\mathrm{TH}^{+}$neurons in zebrafish brain. Data are expressed as a percentage of the control group. Each bar represents mean $\pm \mathrm{SEM} .{ }^{++} \mathrm{p}<0.001$ vs. the control group; ${ }^{*} \mathrm{p}<0.05$ vs. and ${ }^{* *} \mathrm{p}<0.01$ vs. the 6-OHDA-treated group. 
(A)

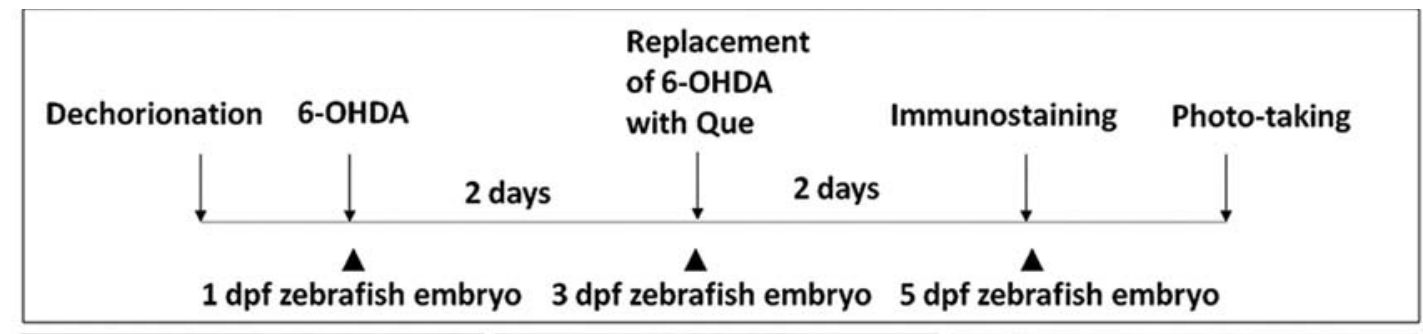

(B)
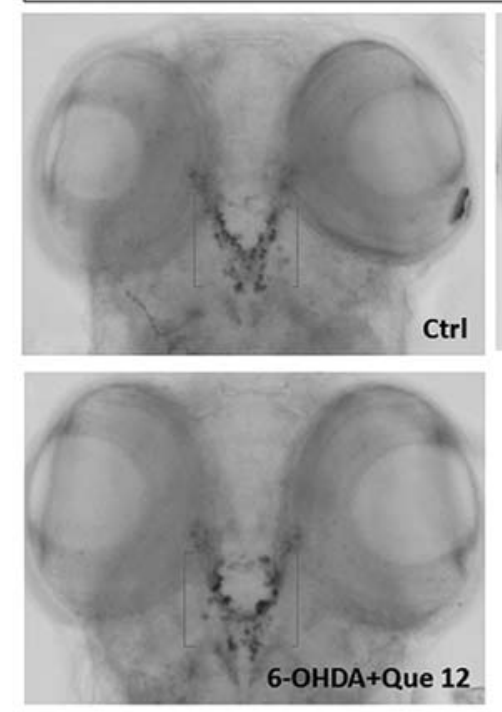
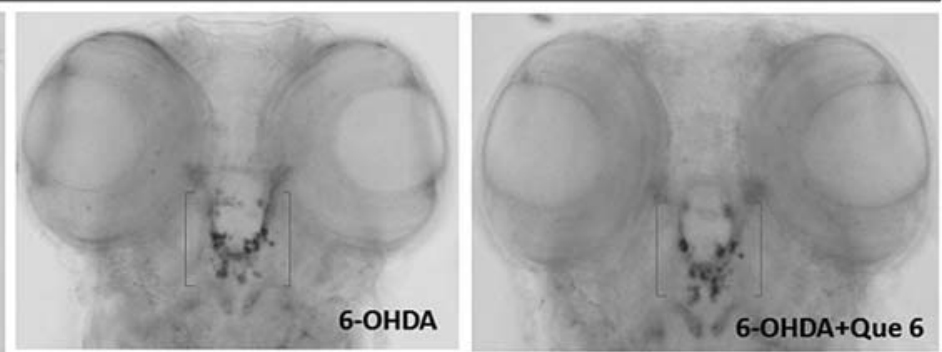

(c)

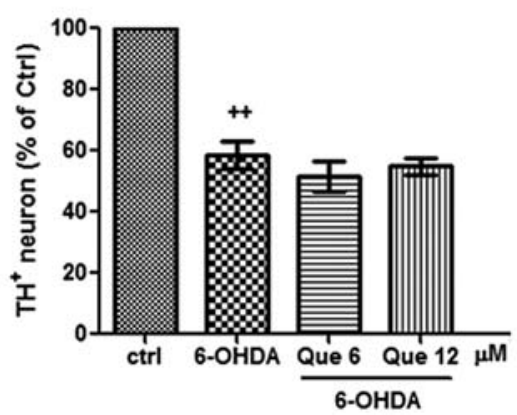

Figure 6. Quercetin (Que) did not rescue DA neuron loss post-6-hydroxydopamine (6-OHDA) treatment in zebrafish. (A) A schematic illustration of drug treatment. Dechorionated $1 \mathrm{dpf}$ zebrafish were treated for $48 \mathrm{~h}$ with $250 \mu \mathrm{M}$ 6-OHDA, then 6-OHDA was replaced with Que and treatment was continued for another $48 \mathrm{~h}$. (B) Representative morphology of diencephalic DA neurons (Di DA) in zebrafish brain, indicated by brackets. (C) Quantitative analysis of tyrosine hydroxylase $(\mathrm{TH})^{+}$neurons in zebrafish brain. Data are expressed as a percentage of the control (Ctrl) value. Each bar represents mean \pm SEM. ${ }^{++} \mathrm{p}<0.001$ vs. the control group.
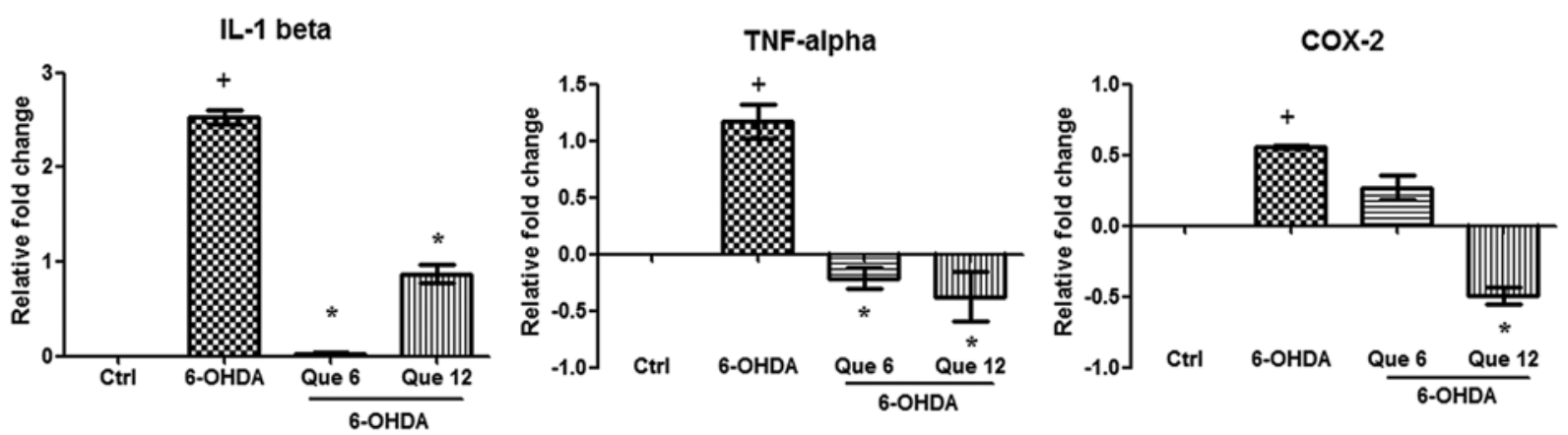

Figure 7. Quercetin (Que) down-regulates pro-inflammatory gene over-expression in zebrafish stimulated by 6-hydroxydopamine (6-OHDA). Zebrafish embryos ( $2 \mathrm{dpf}$ ) were treated for $24 \mathrm{~h}$ with 6-OHDA in the absence or presence of different concentrations of Que. After treatment, mRNA was extracted from each group; quantitative real-time PCR analysis of pro-inflammatory genes (IL-1ß, TNF- $\alpha$ and COX-2) was performed. The results are expressed as the fold-change calculated by the relative $\mathrm{Ct}$ method using $\mathrm{B}$-actin as the internal reference. Each bar represents mean $\pm \mathrm{SEM}$. ${ }^{+} \mathrm{p}<0.05 \mathrm{vs}$. the control $(\mathrm{Ctrl})$ group; ${ }^{*} \mathrm{p}<0.05$ vs. the 6-OHDA-treated group.

in PC12 cells and by the inhibiting inflammatory genes in zerbrafish, respectively. To the best of our knowledge, this is the first study to demonstrate the neuroprotective and antiinflammatory action of quercetin in zebrafish.

Our in vitro study showed that pretreatment with quercetin significantly protected PC12 cells against 6-OHDA neurotoxicity (Figs. 1 and 2), which is in accord with earlier reports that quercetin has a protective effect against toxicity induced by $\mathrm{MPP}^{+}$(36) and $\mathrm{H}_{2} \mathrm{O}_{2}$ (37) in $\mathrm{PC} 12$ cells. However, it has been reported that quercetin alone caused apoptosis and/or cell death in various cell types, including cancer (9) and neuronal SH-SY5Y (38) cells. In PC12 cells, rather than inhibiting, quercetin enhanced $\mathrm{H}_{2} \mathrm{O}_{2}$-induced $\mathrm{PC} 12$ cell death (39). Some effects of quercetin related to enhanced cell death are mediated by mechanisms that require longer drug 
treatment to be manifested, such as by inhibiting heat shock protein 70 expression (40) or reducing the activation of the phosphoinositide 3-kinase pathway (41). A recent report illuminates a dual effect of quercetin from another point of view: quercetin might well be neuroprotective, but it has toxic metabolites that counteract this activity (42). It seems evident that quercetin regulates cell survival and cell death in different ways. For example, the protective effect of quercetin might be due to its anti-oxidative and ROS-scavenging properties, which would take place within a few minutes after the exposure of cells to 6-OHDA, but the toxic effects of the drug need more time to develop.

6-OHDA is selectively taken up by the plasma membrane dopamine transporter and subsequently accumulates in the mitochondria (43). Exposure to 6-OHDA results in the formation of ROS and RNS. It has been demonstrated that the oxidative damage induced by 6-OHDA could be prevented by treatment with antioxidants, such as melatonin $(44,45)$. The results of the present study demonstrate that exposure to 6-OHDA significantly increases NO production and iNOS expression in PC12 cells. LN, a non-specific iNOS inhibitor, showed a neuroprotective effect in the 6-OHDA-induced cell damage in PC12 cells (Figs. 3 and 4). Since quercetin reduced NO over-production and down-regulated the overexpression of iNOS induced by 6-OHDA in PC12 cells, this finding suggests that quercetin exerts its neuro-protective effect through the iNOS-NO pathway.

This gene expression study in zebrafish (Fig. 7) indicated that 6-OHDA up-regulated expression of pro-inflammatory genes, such as IL-1ß, TNF- $\alpha$ and COX-2. IL- $1 \beta$ and TNF- $\alpha$ are important pro-inflammatory cytokines, and COX-2 is a pro-inflammatory enzyme that mediates the production of prostaglandins; they all play important roles in the etiology of Parkinson's disease (46). It has been demonstrated that COX-2 is up-regulated in substantia nigra DA neurons in PD patients and in animal models (47). The inhibition of COX-2 and TNF- $\alpha$ has provided neuroprotection in rats (46). In this study, co-treatment with quercetin significantly suppressed pro-inflammatory gene over-expression stimulated by 6-OHDA. Thus, the neuroprotective effect of quercetin in the 6-OHDA-induced zebrafish model could be explained, at least in part, by its down-regulation of pro-inflammatory gene expression.

The in vivo neuroprotective effect of quercetin has been controversial for decades (17). Several earlier studies found that quercetin has no consistent neuroprotective effect in rat models of 6-OHDA-induced Parkinson's disease $(18,38,48)$. In contrast, some studies showed that quercetin has beneficial effects in various in vivo models of cerebrovascular insults, possibly due to the increased permeability of the BBB in these animal models (49), which can facilitate delivery of quercetin into brain. In addition, treatment of permanent focal ischemia with a lecithin/quercetin preparation decreased lesion volume, showing that this pharmaceutical preparation dosage form could help quercetin to cross the $\mathrm{BBB}$ in vivo (37). These studies show that quercetin itself might cross the BBB ineffectively, which could possibly be enhanced by modified pharmaceutical formulation. Our in vivo study in zebrafish shows that when quercetin is administered at different maturation stages of the BBB in zebrafish it can prevent, but not rescue, the 6-OHDA-induced DA neuron loss (Figs. 5 and 6). This phenomenon could be attributed to the poor permeability of quercetin across the BBB. The zebrafish is a recognized model for the analysis of basic developmental processes, and earlier studies demonstrated that the BBB of zebrafish is present by $72 \mathrm{hpf}(28,50,51)$. Thus, when quercetin was administered to zebrafish larvae before $3 \mathrm{dpf}$, it could penetrate the brain rapidly and exert a protective effect against 6-OHDA toxicity because the BBB was not yet functionally well-developed. However, when quercetin was administered to zebrafish after $3 \mathrm{dpf}$, the matured BBB could pose an obstacle to quercetin reaching the brain, preventing it from rescuing the 6-OHDA-induced dopaminergic insult.

In conclusion, the results from the in vitro and in vivo assays in this study demonstrate the neuro-protective effects of quercetin and add insight into its mechanism of action in 6-OHDA-induced cytotoxicity and neurotoxicity. We have shown that both the iNOS-NO inhibitory and the antiinflammatory actions of quercetin play key roles in the neuroprotective effect of quercetin. Also, the in vivo zebrafish data reported here demonstrate that quercetin can prevent, but not rescue the DA neuron loss induced by 6-OHDA at different maturation stages of the zebrafish BBB. Furthermore, the data provide insight into the importance of $\mathrm{BBB}$ permeability in the manifestation of the beneficial effects of quercetin in $\mathrm{PD}$ in vivo.

\section{Acknowledgements}

This study was supported by grants from the Science and Technology Development Fund of Macau SAR (Ref. No. 045/2007 and 058/2009) and the Research Committee of the University of Macau (Ref. No.UL017/09-Y1).

\section{References}

1. Song L, Song W and Schipper HM: Astroglia overexpressing heme oxygenase- 1 predispose co-cultured PC12 cells to oxidative injury. J Neurosci Res 85: 2186-2195, 2007.

2. Hanrott K, Gudmunsen L, O'Neill MJ and Wonnacott S: 6-hydroxydopamine-induced apoptosis is mediated via extracellular auto-oxidation and caspase 3-dependent activation of protein kinase Cdelta. J Biol Chem 281: 5373-5382, 2006.

3. Hirsch EC, Hunot S and Hartmann A: Neuroinflammatory processes in Parkinson's disease. Parkinsonism Relat Disord 11 (Suppl. 1): S9-S15, 2005.

4. Vila M, Jackson-Lewis V, Vukosavic S, et al: Bax ablation prevents dopaminergic neurodegeneration in the 1-methyl-4phenyl-1,2,3,6-tetrahydropyridine mouse model of Parkinson's disease. Proc Natl Acad Sci USA 98: 2837-2842, 2001.

5. Stewart VC and Heales SJ: Nitric oxide-induced mitochondrial dysfunction: implications for neurodegeneration. Free Radic Biol Med 34: 287-303, 2003.

6. Kozuka N, Itofusa R, Kudo Y and Morita M: Lipopolysaccharide and proinflammatory cytokines require different astrocyte states to induce nitric oxide production. J Neurosci Res 82: 717-728, 2005.

7. Bureau G, Longpre F and Martinoli MG: Resveratrol and quercetin, two natural polyphenols, reduce apoptotic neuronal cell death induced by neuroinflammation. J Neurosci Res 86: 403-410, 2008.

8. Mercer LD, Kelly BL, Horne MK and Beart PM: Dietary polyphenols protect dopamine neurons from oxidative insults and apoptosis: investigations in primary rat mesencephalic cultures. Biochem Pharmacol 69: 339-345, 2005.

9. Shan BE, Wang MX and Li RQ: Quercetin inhibit human SW480 colon cancer growth in association with inhibition of cyclin D1 and survivin expression through Wnt/beta-catenin signaling pathway. Cancer Invest 27: 604-612, 2009. 
10. Kaur G, Roberti M, Raul F and Pendurthi UR: Suppression of human monocyte tissue factor induction by red wine phenolics and synthetic derivatives of resveratrol. Thromb Res 119: 247-256, 2007.

11. Ruiz PA, Braune A, Holzlwimmer G, Quintanilla-Fend L and Haller D: Quercetin inhibits TNF-induced NF-kappaB transcription factor recruitment to proinflammatory gene promoters in murine intestinal epithelial cells. J Nutr 137: 1208-1215, 2007.

12. Choi YJ, Jeong YJ, Lee YJ, Kwon HM and Kang YH: (-)Epigallocatechin gallate and quercetin enhance survival signaling in response to oxidant-induced human endothelial apoptosis. J Nutr 135: 707-713, 2005.

13. Bournival J, Quessy P and Martinoli MG: Protective effects of resveratrol and quercetin against $\mathrm{MPP}^{+}$-induced oxidative stress act by modulating markers of apoptotic death in dopaminergic neurons. Cell Mol Neurobiol 29: 1169-1180, 2009.

14. Ansari MA, Abdul HM, Joshi G, Opii WO and Butterfield DA: Protective effect of quercetin in primary neurons against Abeta(1-42): relevance to Alzheimer's disease. J Nutr Biochem 20: 269-275, 2009

15. Rivera F, Costa G, Abin A, et al: Reduction of ischemic brain damage and increase of glutathione by a liposomal preparation of quercetin in permanent focal ischemia in rats. Neurotox Res 13: $105-114,2008$.

16. Pu F, Mishima K, Irie K, et al: Neuroprotective effects of quercetin and rutin on spatial memory impairment in an 8-arm radial maze task and neuronal death induced by repeated cerebral ischemia in rats. J Pharmacol Sci 104: 329-334, 2007.

17. Ossola B, Kaariainen TM and Mannisto PT: The multiple faces of quercetin in neuroprotection. Expert Opin Drug Saf 8: 397-409, 2009.

18. Dajas F, Costa G, Abin-Carriquiry JA, McGregor R and Urbanavicius $\mathrm{J}$ : Involvement of nicotinic acetylcholine receptors in the protection of dopamine terminals in experimental parkinsonism. Funct Neurol 16 (Suppl. 4): 113-123, 2001.

19. Langheinrich $\mathrm{U}$ : Zebrafish: a new model on the pharmaceutical catwalk. Bioessays 25: 904-912, 2003.

20. Crawford AD, Esguerra CV and De Witte PA: Fishing for drugs from nature: zebrafish as a technology platform for natural product discovery. Planta Med 74: 624-632, 2008.

21. Norrby K: In vivo models of angiogenesis. J Cell Mol Med 10: 588-612, 2006

22. Guo S, Wilson SW, Cooke S, Chitnis AB, Driever W and Rosenthal A: Mutations in the zebrafish unmask shared regulatory pathways controlling the development of catecholaminergic neurons. Dev Biol 208: 473-487, 1999.

23. Rink E and Wullimann MF: The teleostean (zebrafish) dopaminergic system ascending to the subpallium (striatum) is located in the basal diencephalon (posterior tuberculum). Brain Res 889: 316-330, 2001

24. Anichtchik OV, Kaslin J, Peitsaro N, Scheinin M and Panula P: Neurochemical and behavioural changes in zebrafish Danio rerio after systemic administration of 6-hydroxydopamine and 1-methyl-4-phenyl-1,2,3,6-tetrahydropyridine. J Neurochem 88: 443-453, 2004.

25. Bretaud S, Lee S and Guo S: Sensitivity of zebrafish to environmental toxins implicated in Parkinson's disease. Neurotoxicol Teratol 26: 857-864, 2004.

26. Parng C, Roy NM, Ton C, Lin Y and McGrath P: Neurotoxicity assessment using zebrafish. J Pharmacol Toxicol Methods 55: 103-112, 2007.

27. McKinley ET, Baranowski TC, Blavo DO, Cato C, Doan TN and Rubinstein AL: Neuroprotection of MPTP-induced toxicity in zebrafish dopaminergic neurons. Brain Res Mol Brain Res 141: 128-137, 2005.

28. Parng C, Ton C, Lin YX, Roy NM and McGrath P: A zebrafish assay for identifying neuroprotectants in vivo. Neurotoxicol Teratol 28: 509-516, 2006.

29. Bitzur S, Kam Z and Geiger B: Structure and distribution of $\mathrm{N}$-cadherin in developing zebrafish embryos: morphogenetic effects of ectopic over-expression. Dev Dyn 201: 121-136, 1994.
30. Livak KJ and Schmittgen TD: Analysis of relative gene expression data using real-time quantitative PCR and the 2(-Delta Delta $\mathrm{C}(\mathrm{T}))$ method. Methods 25: 402-408, 2001.

31. Woodgate A, MacGibbon G, Walton M and Dragunow M: The toxicity of 6-hydroxydopamine on PC12 and P19 cells. Brain Res Mol Brain Res 69: 84-92, 1999.

32. Shih YT, Chen IJ, Wu YC and Lo YC: San-Huang-Xie-XinTang protects against activated microglia- and 6-OHDAinduced toxicity in neuronal SH-SY5Y cells. Evid Based Complement Alternat Med: April 1, 2009 (Epub ahead of print).

33. Lin YC, Uang HW, Lin RJ, Chen IJ and Lo YC: Neuroprotective effects of glyceryl nonivamide against microglia-like cells and 6-hydroxydopamine-induced neurotoxicity in SH-SY5Y human dopaminergic neuroblastoma cells. J Pharmacol Exp Ther 323: 877-887, 2007.

34. Singh S, Das T, Ravindran A, et al: Involvement of nitric oxide in neurodegeneration: a study on the experimental models of Parkinson's disease. Redox Rep 10: 103-109, 2005.

35. Cicchetti F, Brownell AL, Williams K, Chen YI, Livni E and Isacson O: Neuroinflammation of the nigrostriatal pathway during progressive 6-OHDA dopamine degeneration in rats monitored by immunohistochemistry and PET imaging. Eur J Neurosci 15: 991-998, 2002.

36. Gelinas S and Martinoli MG: Neuroprotective effect of estradiol and phytoestrogens on $\mathrm{MPP}^{+}$-induced cytotoxicity in neuronal PC12 cells. J Neurosci Res 70: 90-96, 2002.

37. Dajas F, Rivera-Megret F, Blasina F, et al: Neuroprotection by flavonoids. Braz J Med Biol Res 36: 1613-1620, 2003.

38. Kaariainen TM, Piltonen M, Ossola B, et al: Lack of robust protective effect of quercetin in two types of 6-hydroxydopamineinduced parkinsonian models in rats and dopaminergic cell cultures. Brain Res 1203: 149-159, 2008.

39. Sasaki M, Nakamura H, Tsuchiya S, et al: Quercetin-induced PC12 cell death accompanied by caspase-mediated DNA fragmentation. Biol Pharm Bull 30: 682-686, 2007.

40. Hosokawa N, Hirayoshi K, Nakai A, et al: Flavonoids inhibit the expression of heat shock proteins. Cell Struct Funct 15: 393-401, 1990.

41. Walker EH, Pacold ME, Perisic O, et al: Structural determinants of phosphoinositide 3-kinase inhibition by wortmannin, LY294002, quercetin, myricetin, and staurosporine. Mol Cell 6: 909-919, 2000.

42. Boots AW, Li H, Schins RP, et al: The quercetin paradox. Toxicol Appl Pharmacol 222: 89-96, 2007.

43. Blum D, Torch S, Lambeng N, et al: Molecular pathways involved in the neurotoxicity of 6-OHDA, dopamine and MPTP: contribution to the apoptotic theory in Parkinson's disease. Prog Neurobiol 65: 135-172, 2001.

44. Aguiar LM, Vasconcelos SM, Sousa FC and Viana GS: Melatonin reverses neurochemical alterations induced by 6-OHDA in rat striatum. Life Sci 70: 1041-1051, 2002.

45. Sharma R, McMillan CR, Tenn CC and Niles LP: Physiological neuroprotection by melatonin in a 6-hydroxydopamine model of Parkinson's disease. Brain Res 1068: 230-236, 2006.

46. Hunter RL, Dragicevic N, Seifert K, et al: Inflammation induces mitochondrial dysfunction and dopaminergic neurodegeneration in the nigrostriatal system. J Neurochem 100: 1375-1386, 2007.

47. Esposito E, Di Matteo V, Benigno A, Pierucci M, Crescimanno G and Di Giovanni G: Non-steroidal anti-inflammatory drugs in Parkinson's disease. Exp Neurol 205: 295-312, 2007.

48. Costa G, Abin-Carriquiry JA and Dajas F: Nicotine prevents striatal dopamine loss produced by 6-hydroxydopamine lesion in the substantia nigra. Brain Res 888: 336-342, 2001.

49. Huber JD, Egleton RD and Davis TP: Molecular physiology and pathophysiology of tight junctions in the blood-brain barrier. Trends Neurosci 24: 719-725, 2001.

50. Jeong JY, Kwon HB, Ahn JC, et al: Functional and developmental analysis of the blood-brain barrier in zebrafish. Brain Res Bull 75: 619-628, 2008.

51. Garcia-Lecea M, Kondrychyn I, Fong SH, Ye ZR and Korzh V: In vivo analysis of choroid plexus morphogenesis in zebrafish. PLoS One 3: e3090, 2008. 